\title{
A Contingency Approach to Enterprise Architecture Method Engineering
}

\author{
Christian Riege and Stephan Aier \\ Institute of Information Management, University of St. Gallen, \\ Müller-Friedberg-Strasse 8 \\ 9000 St. Gallen, Switzerland \\ \{christian.riege, stephan.aier\}@unisg.ch
}

\begin{abstract}
Enterprise Architecture (EA) and methods for the design and employment of EA significantly contribute to the transparency, consistency and eventually to the flexibility of an organization. However, there is hardly any "one-size-fits-all" EA method that is equally effective for a broad range of transformation projects or in a large number of different contexts. Based on an empirical analysis, this paper identifies three relevant EA contingency factors as well as three dominating EA application scenarios as a basis for a situational EA method engineering taking these into account.
\end{abstract}

Keywords: Enterprise Architecture, Method Engineering, Contingency Factors.

\section{Introduction}

Enterprise architecture (EA) describes the fundamental structure of an enterprise [28, 33] and supports transformation by offering a holistic perspective on as-is as well as to-be structures and processes [19].

EA is widely accepted as an approach to manage transformations and to foster IT/business alignment [7, 22, 30]. In order to guide this transformation, EA methods are needed. While there are a number of EA methods available, e.g. [21, 31], a classification of methods is needed in order to understand in which situation a certain method is appropriate, how a method should be adapted to a certain situation, or for which situations new methods have to be developed. This is based on the assumption that there is no "one-size-fits-all" method, but that, depending on a certain situation, different methods - or at least different configurations or adaptations of a methodare needed. In order to develop an understanding of such situations, relevant contingency factors need to be identified which have an impact on the realization of EA.

As a foundation for situational EA method engineering and to continue the discussion started in [1] this paper further particularizes current realization approaches of EA. EA method engineering is an evolving discipline, which additionally requires outlining typical EA application scenarios. For this purpose our contribution is based on an exploratory analysis shaping current EA approaches, EA contingency factors as well as important EA application scenarios.

The remainder of this paper is organized as follows: Section two provides an overview of the theoretical background and related work. The discussion of the 
contingency factors in situational method engineering is reflected, and a short review on the state-of-the-art of enterprise architecture is given. Section three describes the details of the explorative empirical analysis aiming at identifying current EA realization approaches and section four outlines typical EA application scenarios. The paper ends with a conclusion and an outlook on future research activities in section five.

\section{Theoretical Background and Related Work}

Enterprise architecture literature provides a broad range of results that may be grouped into three categories. Category one comprises enterprise architecture frameworks. Popular examples are the Zachman Framework [35] and The Open Group Enterprise Architecture Framework-TOGAF [28]. Category two is comprised of publications by scientists as for example [8, 14, 19]. The third category is defined by practitioner's publications who predominantly publish for practitioners. Examples are $[24,29]$. However, the boundary between scientific and practitioner approaches (regarding authorship as well as readership) is often fluid. Examples are [3, 22, 27].

A fundamental method provided by almost all of the contributions cited above is comprised of a basic approach for documenting the as-is and/or to-be EA. Some approaches provide a number of analyses that may be employed in an EA transformation method $[19,20]$ or a list of EA application scenarios for which methods may be developed. However, this list is neither complete nor are its items disjunctive.

Discussion in the field of EA is highly concerned with questions as which artefacts belong to EA, e.g. [2, 4, 15]. Only recently, it is discussed how to maintain EA models [7], how to use EA, or what benefits EA may provide to an organization [25]. Especially the latter issues require sound methods. Although there are isolated EA methods taking the situation of application into account, e.g. [34], there is no overall landscape of EA methods available.

A method may be defined as a systematic aid that guides the transformation of a system from an initial state to a target state. It is unlikely that there is an EA method, which fits to every problem situation in the field. Instead it is advisable to adapt an existing method or to use dedicated parts, like method components or method fragments. Approaches like this are discussed as situational method engineering $[12,18$, 26]. It means that a method can be customized for the needs of a project or an organisation. In order to customize a method for a situation contingency or situational factors are needed to facilitate the description of such a situation. Existing contingency approaches are not tailored for EA and their contingency factors often lack empirical evidence $[13,17,26]$. Therefore the aim of this paper is to identify contingency factors determining current EA realization approaches by means of empirical analysis.

\section{Current Realization Approaches of EA}

An exploratory analysis was conducted in order to identify different EA approaches in practice [1]. The data was collected by means of a questionnaire filled in by participants of two practitioner conferences in 2007. Both conferences focused on EA in particular. Attending were IT executives, IT service providers and consultants as well as EA experts. In advance of the conferences, the questionnaire was subject to a pretest carried out by a group of enterprise architects. 


\subsection{Characteristics of the Data Set}

A total of 69 questionnaires were returned. If the data set was incomplete regarding one of the 15 items used in subsequent analysis, the questionnaire was discarded. After applying this selection criterion, 55 valid questionnaires were analyzed. Although the sample size is rather small, the data set is considered adequate to provide a basis for an exploratory analysis. ${ }^{1}$ The observed organizations mainly represent mid-size and large companies from the financial services sector as well as software vendors and IT consultants. In addition to demographic characteristics the data set comprises variables which can be divided into four groups and characterized as follows:

Constitution of EA: Architecture in general includes a set of IT artefacts like hardware assets, software components, and applications and extends the focus to business related artefacts. To ensure that business/IT alignment is adequately supported, EA also spans artefacts like business processes, products, customer segments, etc. Due to the large number of potential artefacts, EA is requested to represent the essential parts of the organization [35]. The data set contains information regarding the aforementioned variables.

Application scenarios and analysis techniques of EA: The employment of EA in an organization often refers to a substantial number of possible applications [19, 20, 32]. Applications however are external to the EA approach. The aim is to integrate EA into the organization's initiatives to secure that the organization develops in accordance with the structures defined in EA. For this reason, the EA model is subject to a range of analysis techniques. Techniques reveal dependencies between different EA artefacts, identify gaps or redundancies (e.g. application support of certain business processes), and reveal artefacts that might interfere with a homogeneous EA structure $[19,20,32]$.

Maintenance of EA: This part of the data set contains information to which extent EA models are part of strategic planning, and to which extent EA models support transformations. Furthermore it covers the approach how EA data is gathered and maintained within an organization. A central instance for EA-related information facilitates a less complex and consistent EA improvement. In this holistic approach, a "leading" EA model is maintained covering all artefacts used to describe the EA. A federated approach puts more emphasis on specialized architectures and their models. The EA model is then supplied with data through periodically performed replications. EA data which is maintained via local repositories yields more flexibility, but also ensures that the stored information is up-to-date [7].

Communication and organizational structure of EA: On the one hand, the data set contains information on organizational roles which should be established to ensure EA is adequately represented within the organization-e.g. the role of an expert in EA modelling. On the other hand, EA offers benefits that take effect across IT and business units. It is important to capture how the concept of EA spreads within the organization. According to the understanding that EA is also involved in management

\footnotetext{
${ }^{1}$ What rule of thumb to use for factor analysis in determining an allowable ratio of cases to variables is still a matter of research taste [23]. However, [5] suggests a 4-to-1 rule of thumb for an allowable ratio of cases to variables.
} 
activities and addresses business related objectives, it is of high importance how EA is perceived [14]. The information in this part of the questionnaire also covers the integration of EA processes into the organization's governance structure. The respondents were asked to assess the current degree of realization of each item in their organization. Therefore, the questionnaire chooses a five-tiered Likert scale. The minimum value (1) that was possible to check represents "nonexistent", whereas the maximum value (5) indicates an "optimized" realization.

\subsection{Identifying Contingency Factors of EA}

In order to identify contingency factors of EA, a factor analysis is applied. A factor analysis involves extracting a small number of latent factors among the variables in the data set. To form an adequate foundation, the data set has to meet two criteria. The first criterion is derived from the variables' anti image covariance. The anti image covers the part of the variance which cannot be explained by the remaining variables in the data set. As factor analysis aims at finding latent factors based on the data set, a data set is suitable for factor analysis if the anti image is rather low. According to [6], the percentage of none diagonal elements of the anti image covariance matrix, which are non-zero $(>0.09)$, should not exceed $25 \%$. In the case presented here, this parameter is about $17 \%$. The second criterion involves the computation of the Kaiser-MeyerOlkin measure of sampling adequacy. In the data set at hand, the measure is 0.798 . According to [16], it puts a data set with a value of 0.7 or above into "middling" range, bordering the "meritorious" range. In this case, the results proof that the data set is generally appropriate for factor analysis. The factor analysis was performed based on a reduced data set of 15 items. While some items which are excluded from subsequent analyses relate to company properties such as staff size and industry sector, others were previously characterized as covering the constitution of EA within an organization.

As extraction method the principal component analysis was applied. Principal component analysis identifies few independent factors that contain the fundamental aspects of the data. In order to identify the optimum number of factors, the eigenvalue was computed which represents the amount of variance accounted for by a factor. According to the Kaiser criterion a factor's eigenvalue should exceed a value of one [10]. As a result, three contingency factors that account for $64 \%$ of the total variance were extracted. In order to better interpret the nature of the factors, the component matrix was rotated applying the Varimax method with Kaiser normalization. Each of the three factors consists of five items and can be described as follows.

Table 1. Factor 1-Adoption of advanced architectural design paradigms and modelling capabilities

Item 1.1 EA is developed with regard to modularization as an architectural design paradigm.

Item 1.2 The principles of service orientation form a basis on which EA is designed.

Item 1.3 EA models represent the current structure of the organization.

Item 1.4 Documentation of EA models includes target architecture.

Item 1.5 EA models support transforming EA from as-is structure towards to-be structures. 
The items that load on contingency factor 1 describe valuable ways to adopt the concept of EA. On the one hand, it involves well established architecture design paradigms which emphasize the layered structure of EA. The findings denote that developing EA needs a certain degree of decoupling between the different EA layers as indicated by the principles of service orientation and thus foster re-use of EA artefacts. On the other hand, factor 1 points out that a further enhancement of EA also depends on the dimension of the EA documentation. To allow for a continuous development, not only loosely coupled artefacts, but also an idea of how to approach a future development stage is decisive. EA then contributes to business/IT alignment by offering simulation capabilities, which presupposes different variants of its to-be structures.

Table 2. Factor 2-Deployment and monitoring of EA data and services

Item 2.1 EA is measured and/or reviewed on a regular basis.

Item 2.2 Processes concerning EA management are subject to regular reviews.

Item 2.3 The role of an EA quality manager is established fostering and communicating EA concerns.

Item 2.4 EA is aiming to improve the overall homogeneity of architecture elements by applying heterogeneity analysis.

Item 2.5 EA is used to perform coverage analysis in order to illustrate redundancies or gaps regarding EA artefacts.

Factor 2 describes the deployment of EA within the organization. It is required to establish a consistent monitoring of EA data and services to further enforce the deployment. This can be assisted by the role of an EA quality manager who is responsible for observing periodic reviews of EA data and EA processes. A high degree of EA deployment puts the organization in the position to reduce its costs for maintenance activities, software and hardware licenses, but also to ensure that similar concerns are treated equally and according to the parameters of the EA roadmap. A high factor value also points to the application of sophisticated EA analysis techniques within the organization.

Table 3. Factor 3-Organizational penetration of EA

Item 3.1 EA is perceived as being valuable to the business units.

Item 3.2 IT departments explicitly refer to EA as a helpful instrument.

Item 3.3 IT departments use EA data in broad range of use cases.

Item 3.4 Business units base their work on EA data.

Item 3.5 EA data is part of the decision support for management units.

The third contingency factor accounts for the penetration of EA in the organization. The findings suggest that the overall level of penetration is influenced by the degree EA results and EA documentation are used by a broad range of stakeholders. According to this analysis, EA is a suitable tool not only to support IT related work, but also to serve the business units and to provide reliable information to management units. The findings suggest that as the level of organizational penetration increases with the organization's capability to clearly communicate EA benefits to the potential stakeholders-regardless if they actually operate on EA results or not. Therefore, the third factor describes the way EA is perceived and utilized across the organization. A high 
level of organizational penetration leads to a higher acceptance, and less misinterpretation of EA within the organization, respectively.

\subsection{Clustering EA Realization Approaches}

In order to point out how EA is actually realized, the data set was partitioned into different subsets by means of a hierarchical cluster analysis. As input data, the factor values of the three aforementioned contingency factors were used. Ward's method has been used as clustering algorithm. It combines the two clusters which lead to a minimal increase in the within-cluster sum of squares with respect to all variables across all clusters. The squared Euclidean distance was selected as distance measure to determine the similarity of two clusters. Although the application of alternative measures may lead to different clustering results, the squared Euclidean distance was chosen as it is the most commonly recognized procedure [10] and moreover provides a comprehensible representation with respect to the sample's data structure. To gain information about the cohesiveness of clusters, a tree diagram — designated as dendrogram — serves as visualization and helps to assess the appropriate number of clusters to keep. There is no standard selection procedure to derive the number of clusters [10]. As the applied fusion algorithm aims at minimizing the within-cluster sum of squares in each step, it is appropriate to keep the number of clusters if the subsequent clustering step accounts for the highest increase of the total sum of squares [9]. In the analysis at hand, this heuristic suggests to distinguish between three clusters which in turn represent three different EA approaches. Table 4 exhibits arithmetic means $(\bar{x})$ and sample standard deviations $(S)$ of the calculated factor values for each of the three clusters. A high value implies a high degree of realization among the cluster members regarding the factor items that load on the respective factor.

Table 4. Arithmetic mean and standard deviation of factor values

\begin{tabular}{lrrrrrr}
\hline & \multicolumn{2}{c}{ Contingency Factor 1 } & \multicolumn{2}{r}{ Contingency Factor 2 } & \multicolumn{2}{r}{ Contingency Factor 3 } \\
\hline & $\bar{x}$ & $S$ & $\bar{x}$ & $s$ & $\bar{x}$ & $s$ \\
\hline Cluster 1 $(n=15)$ & 1.24 & 0.74 & 0.26 & 1.11 & 0.29 & 0.95 \\
Cluster 2 $(n=10)$ & -0.20 & 0.83 & 0.62 & 1.26 & -1.33 & 0.53 \\
Cluster 3 $(n=30)$ & -0.55 & 0.51 & -0.34 & 0.70 & 0.30 & 0.77 \\
\hline
\end{tabular}

Based on the information depicted in Table 4, the three clusters can be visualized by positioning them in a three dimensional coordinate system (Fig. 1). The horizontal axis of the coordinate system is represented by the factor adoption of advanced architectural design paradigms and modelling capabilities, the vertical axis displays factor 3 organizational penetration of EA. The Factor deployment and monitoring of EA data and services spans the third dimension. The clusters are arranged according to their arithmetic mean (cf. Table 4). To estimate the mean of the population when the sample size is small it is suggested to calculate the confidence interval that is derived from the Student's t-distribution [11]. For this purpose the confidence interval was calculated for each cluster based on the respective mean factor values (cf. Fig. 1). As a result the three cuboids visualize that each cluster differs significantly from another cluster in at least one dimension. 


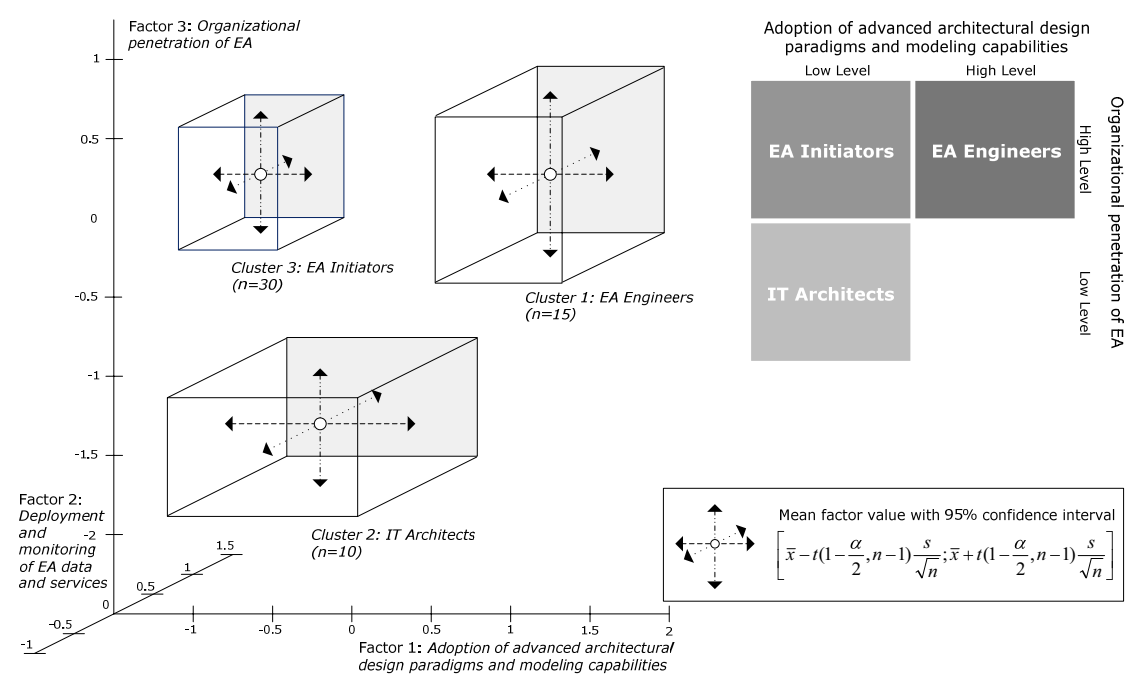

Fig. 1. Enterprise architecture realization approaches

Fig. 1 also exhibits the corresponding two-dimensional classification matrix, which excludes factor 2 as it does not account for significant cluster distinction. The matrix illustrates the distinct EA realization approaches considering factors 1 and 3. For both dimensions, high and low level are distinguished, which refer to either high or low parameter values. The realization approaches of EA can be characterized as follows:

Cluster 1: All 15 organizations which are assigned to this cluster, are characterized by sophisticated implementation of architectural design paradigms. They understand EA as instrument to represent a current structure of the organization, but also to deliver a roadmap for a future structure. It is reasonable to assume that organizational penetration is rather advanced among the members of the cluster. They are using EA rather as IT instrument, but also as a means of communication with the business. The organizations which belong to this cluster constitute an EA approach which may be designated as "EA Engineers". EA engineers understand EA as a valuable instrument to develop and thus transform EA in its holistic understanding. They can also rely on a progressive perception of EA within the business and management units. EA engineers in its current state have an intermediate maturity regarding the employment and monitoring of EA data and services (factor 2).

Cluster 2: The second cluster is made up of 10 organizations which have a low level of both the organizational penetration of EA and the adoption of advanced architectural design paradigms and modelling capabilities. This combination can be characterized as observant attitude regarding a holistic EA. In this case, EA focuses primarily on IT architecture and, therefore, EA data is basically used in traditional IT project development. The relatively high value regarding the second factor supports this characteristic as it indicates a high deployment of (IT related) EA data. The EA approach represented by the organizations which are merged in the second cluster can be designated as "IT Architects". They are well anchored in the IT domain. However, 
this limited architectural understanding is an obstacle in order to really leverage the value of available IT understanding, models and methods. Rather advanced architectural design paradigms-e.g. service orientation-are not much developed in this cluster because they require a certain amount of organizational penetration.

Cluster 3: A total of 30 organizations are grouped into the third cluster. They are characterized by a high level of organizational penetration of EA-comparable with cluster 1. It is therefore reasonable to assume that the potential benefits of EA are recognized among these organizations. EA is understood not only as IT architecture, but also as an instrument to foster the alignment between IT and business. However, EA primarily focuses on documentation. Organizations which belong to this cluster can be designated as "EA Initiators". EA initiators put emphasis on transparency as the necessary precondition to realize benefits from EA application. Therefore, it seems reasonable to conclude that EA initiators in particular are interested in implementing relevant applications to demonstrate these benefits. This also explains the need for more sophisticated analysis techniques-which EA initiators lack of. This typically is a hint for a tool driven or model driven EA approach as opposed to an application driven approach. Such a tool driven approach may be dangerous since it requires significant efforts to survey and model the architectural data without a clear idea of future application scenarios.

The size of the clusters (Table 4) leads to the assumption that most organizations acknowledge the benefits of EA as EA initiators account for more than $50 \%$ of the three EA scenarios. Still a minority of organizations represented by the cluster IT architects is not able to convince potential stakeholders of EA benefits and thus is not able to leverage advanced design or modelling capabilities. The EA scenario with the currently most mature application of EA is represented by EA engineers.

\section{EA Applications Scenarios}

To adequately support EA method engineering, it is not sufficient to take contingency factors (c.f. section 3) into account but also to describe future EA application scenarios. In order to identify these scenarios, a second factor analysis has been performed based on 12 EA applications. The set of EA applications is derived from [20, 32]. Factor analysis serves as a means to reduce the dimensionality of that number of EA applications to a fewer number of factors. In contrast to the analysis in section 3.1, the respondents were asked to asses the future importance of each of the 12 suggested EA applications. As quality measures, the anti image covariance matrix as well as the Kaiser-Meyer-Olkin criteria were computed. In the data set at hand the percentage of none diagonal elements of the anti image covariance matrix is about $22 \%$, well in range with the limit of $25 \%$ [6]. The Kaiser-Meyer-Olkin measure of sampling adequacy is about 0.828 , putting the data set in the "meritorious" range [16]. The results assure that the data set is appropriate for subsequent factor analysis. Principal component analysis extracts three independent factors, which inherit the aspects of the 12 underlying EA applications. In total the three factors, representing three scenarios for EA application, account for $67.6 \%$ of the variance. Factor 1 consists of 4 items (Table 5) and can be characterized as follows. 
Table 5. Factor 1-Support of Business Strategy Development

\begin{tabular}{ll}
\hline Item 1.1 & Corporate Strategy Planning \\
Item 1.2 & Business Process Optimization \\
Item 1.3 & Quality Management and Improvement \\
Item 1.4 & Business Continuity Planning \\
\hline
\end{tabular}

Factor 1 describes EA applications that affect the strategic development of an organization. EA supports decisions which e. g. demand reengineering business functions due to a potential shift in market requirements like quality aspects or processing time. Strategy development involves further analysis like a feasibility analysis to offer certain product bundles. EA is used to identify market offers/products that will be affected in case a specific application fails. In terms of business process optimization EA is a means to discover redundant processes which contribute to the same market offers/products. As an instrument being supportive for business strategy development, EA will need to revert to certain artefacts within the organisation. It is therefore necessary to have transparency e. g. regarding market segments, product catalogues, and business functions of the organisation and their interdependencies.

Table 6. Factor 2-Support of IT Management

Item $2.1 \quad$ IT Consolidation

Item 2.2 Application Portfolio Management

Item 2.3 IT/Business Alignment

Item 2.4 Adoption of COTS

Item 2.5 Architecture Compliance Assessment

Factor 2 comprises of 5 items, (Table 6). Items which load on factor 2 specifically support IT Management within an organisation. Within this scenario EA is concerned with e. g. the advancement of the application landscape. EA serves as a means for analyzing the lifecycle of applications, and for example evaluate alternative replacement decisions. This is particularly important, if COTS is going to replace existing parts of the application landscape. Furthermore IT Management uses EA as a tool to consolidate the application landscape e. g. by analyzing whether there are applications without assigned users. Regarding IT Project Management, EA documents and provides an overview of compliance concerning technical or platform standards. EA artefacts, which are necessary to facilitate IT Management include applications, software components and hardware assets as well as the project portfolio. In this context EA is understood as a complementing approach to CMDB or IT Project Management.

Table 7. Factor 3-Support of Business Operations

Item 3.1 Security Management

Item 3.2 Sourcing and Business Networking

Item 3.5 Compliance Management 
Factor 3 describes EA applications, which support daily business operations (Table 7). In contrast to factor 1 Support of Business Strategy Development EA is more concerned with maintaining the conditions and quality attributes the business requires to carry out its operations and only to a less extent with long term planning. By e. g. analysing business process roles, their correct embedment within the authorization structure of corresponding applications, EA ensures that the organization's identity management is aligned and consistent with business requirements. To support sourcing decisions and maintain SLA, EA provides transparency regarding process interfaces. This enables the organization to analyze whether such interfaces are compliant to a service provider. In this scenario required artefacts range from business processes, interfaces, and SLA to business networking partners.

\section{Summary and Future Work}

Based on the discussion in situational method engineering and the current EA stateof-the-art, this paper suggests differentiating contingency factors of EA. The results of the exploratory analysis confirm the assumption that there is no overall approach to adapt to EA in practice, but to distinguish between three EA realization approaches. They represent three different approaches on how to grasp EA in terms of its determining factors. The exploratory analysis (Fig. 2) shows that adoption of advanced architectural design paradigms and modelling capabilities, and organizational penetration of EA are significant factors to discriminate between different EA approaches in practice. The fact that EA (as opposed to IT architecture) is a pretty novel topic is addressed by an analysis of possible EA applications. The presented contingency factors, resulting in different realization approaches and application scenarios provide a basis on which EA methods can be adapted to a specific situation.

A possibility to consolidate and validate the findings of the analysis at hand is to build EA methods employing these contingency factors, respectively the EA approaches and application scenarios, and evaluate these methods in real life case studies. This will help to further enhance the construction of methods for an effective EA management, where methods specifically fit to the situations in which they are applied.

Although we do not interpret the identified EA realization approaches as levels of EA maturity, we expect the members of each cluster to further develop their EA. This is an important starting point for further research activities, where the exploration, description, and in particular the methodical support of such transformation or development paths have to be covered.

\section{References}

1. Aier, S., Riege, C., Winter, R.: Classification of Enterprise Architecture Scenarios - An Exploratory Analysis. Enterprise Modelling and Information Systems Architectures 3(1), 14-23 (2008)

2. Arbab, F., de Boer, F., Bonsangue, M., Lankhorst, M., Proper, E., van der Torre, L.: Integrating Architectural Models. Symbolic, Semantic and Subjective Models in Enterprise Architecture. Enterprise Modelling And Information System Architectures 2(1), 40-56 (2007) 
3. Bernard, S.A.: An Introduction to Enterprise Architecture, 2nd edn. Authorhouse, Bloomington (2005)

4. Buckl, S., Ernst, A.M., Lankes, J., Matthes, F., Schweda, C.M., Wittenburg, A.: Generating Visualizations of Enterprise Architectures using Model Transformations. Enterprise Modelling and Information Systems Architectures 2(2), 3-13 (2007)

5. Cattell, R.B.: Factor Analysis: An Introduction and Manual for the Psychologist and Social Scientist. Harper and Row, New York (1952)

6. Dziuban, C.D., Shirkey, E.C.: When is a correlation matrix appropriate for factor analysis? Psychological Bulletin 81(6), 358-361 (1974)

7. Fischer, R., Aier, S., Winter, R.: A Federated Approach to Enterprise Architecture Model Maintenance. Enterprise Modelling and Information Systems Architectures 2(2), 14-22 (2007)

8. Frank, U.: Multi-Perspective Enterprise Modeling (MEMO) - Conceptual Framework and Modeling Languages. In: Proceedings of the Hawaii International Conference on System Sciences (HICSS-35) (2002)

9. Gordon, A.D.: Hierarchical Classification. In: Arabie, P., Hubert, L.J., De Soete, G. (eds.) Clustering and Classification, pp. 65-121. World Scientific Publishing, River Edge (1996)

10. Hair Jr., J.F., Black, B., Babin, B.: Multivariate Data Analysis, 6th edn. Prentice Hall, Englewood Cliffs (2006)

11. Härdle, W., Simar, L.: Applied Multivariate Statistical Analysis. Springer, Berlin (2003)

12. Harmsen, A.F., Brinkkemper, S., Oei, H.: Situational Method Engineering for Information System Project Approaches. In: Proceedings of the IFIP 8.1 Working Conference on Methods and Associated Tools for the Information Systems Life Cycle, pp. 169-194. NorthHolland, Amsterdam (1994)

13. Huisman, M., Iivari, J.: The individual deployment of systems development methodologies. In: Pidduck, A.B., Mylopoulos, J., Woo, C.C., Ozsu, M.T. (eds.) CAiSE 2002. LNCS, vol. 2348, pp. 134-150. Springer, Heidelberg (2002)

14. Johnson, P., Ekstedt, M.: Enterprise Architecture - Models and Analyses for Information Systems Decision Making. Studentlitteratur, Pozkal (2007)

15. Jonkers, H., Lankhorst, M., van Buuren, R., Hoppenbrouwers, S., Bonsangue, M., van der Torre, L.: Concepts for Modelling Enterprise Architectures. International Journal of Cooperative Information Systems 13(3), 257-287 (2004)

16. Kaiser, H.F., Rice, J.: Little Jiffy, Mark IV. Educational and Psychological Measurement 34(1), 111-117 (1974)

17. Kettinger, W.J., Teng, J.T.C., Guha, S.: Business Process Change: A Study of Methodologies, Techniques, and Tools. MISQ 21(1), 55-80 (1997)

18. Kumar, K., Welke, R.J.: Methodology Engineering - A Proposal for Situation-specific Methodology Construction. In: Cotterman, W., Senn, J.A. (eds.) Challenges and Strategies for Research in Systems Development, pp. 257-269. John Wiley \& Sons, New York (1992)

19. Lankhorst, M.: Enterprise Architecture at Work: Modelling, Communication and Analysis. Springer, Berlin (2005)

20. Niemann, K.D.: From Enterprise Architecture to IT Governance. Elements of Effective IT Management. Vieweg, Wiesbaden (2006)

21. Pereira, C.M., Sousa, P.: A Method to Define an Enterprise Architecture using the Zachman Framework. In: Proceedings of the 2004 ACM Symposium On Applied Computing, pp. 1366-1371. ACM Press, New York (2004)

22. Ross, J.W., Weill, P., Robertson, D.C.: Enterprise Architecture as Strategy. Creating a Foundation for Business Execution. Harvard Business School Press, Boston (2006) 
23. Rummel, R.J.: Applied Factor Analysis. Northwestern University Press, Chicago (1970)

24. Schekkerman, J.: How to Survive in the Jungle of Enterprise Architecture Frameworks: Creating or Choosing an Enterprise Architecture Framework, 2nd edn. Trafford Publishing, Victoria (2004)

25. Schelp, J., Stutz, M.: A Balanced Scorecard Approach to Measure the Value of Enterprise Architecture. Journal of Enterprise Architecture 3(4), 8-14 (2007)

26. van Slooten, K., Hodes, B.: Characterizing IS Development Projects. In: Proceedings of the IFIP TC8, WG8.1/8.2 Working Conference on Method Engineering, pp. 29-44. Springer, Berlin (1996)

27. Spewak, S.H., Hill, S.C.: Enterprise Architecture Planning - Developing a Blueprint for Data, Applications and Technology. John Wiley \& Sons, New York (1993)

28. The Open Group, The Open Group Architecture Framework TOGAF - 2007 Edition (Incorporating 8.1.1). Van Haren, Zaltbommel (2007)

29. Theuerkorn, F.: Lightweight Enterprise Architectures. Auerbach Publishers, Boca Raton (2004)

30. Veasey, P.W.: Use of enterprise architectures in managing strategic change. Business Process Management Journal 7(5), 420-436 (2001)

31. Wegmann, A.: The Systemic Enterprise Architecture Methodology (SEAM) - Business and IT Alignment for Competiveness, École Poytechnique Fédérale de Lausanne (2002)

32. Winter, R., Bucher, T., Fischer, R., Kurpjuweit, S.: Analysis and Application Scenarios of Enterprise Architecture - An Exploratory Study. Journal of Enterprise Architecture 3(3), 33-43 (2007)

33. Winter, R., Fischer, R.: Essential Layers, Artifacts, and Dependencies of Enterprise Architecture. Journal of Enterprise Architecture 3(2), 7-18 (2007)

34. Ylimäki, T., Halttunen, V.: Method engineering in practice: A case of applying the Zachman framework in the context of small enterprise architecture oriented projects. Information Knowledge Systems Management 5(3), 189-209 (2006)

35. Zachman, J.A.: A Framework for Information Systems Architecture. IBM Systems Journal 26(3), 276-292 (1987) 